\title{
Existence and Exponential Decay of Solutions to a Quasilinear Thermoelastic Plate System
}

\author{
Irena Lasiecka, Sara Maad and Amol Sasane
}

\begin{abstract}
We consider a quasilinear PDE system which models nonlinear vibrations of a thermoelastic plate defined on a bounded domain in $\mathbb{R}^{n}, n \leq 3$. Existence of finite energy solutions describing the dynamics of a nonlinear thermoelastic plate is established. In addition asymptotic long time behavior of weak solutions is discussed. It is shown that finite energy solutions decay exponentially to zero with the rate depending only on the (finite energy) size of initial conditions. The proofs are based on methods of weak compactness along with nonlocal partial differential operator multipliers which supply the sought after "recovery" inequalities. Regularity of solutions is also discussed by exploiting the underlying analyticity of the linearized semigroup along with a related maximal parabolic regularity $[1,16,44]$.
\end{abstract}

Mathematics Subject Classification (2000). Primary 74F05; Secondary 35B30, 35B40, 74H40.

Keywords. Quasilinear thermoelastic plates, existence of weak solutions, uniform decays of finite energy solutions.

\section{Introduction}

In this paper we study the existence and exponential stability of solutions to a quasilinear system arising in the modeling of nonlinear thermoelastic plates. The mathematical analysis of thermoelastic systems has attracted a lot of attention over the years. An array of new and fundamental results in the area of wellposedness and stability of solutions to both linear and nonlinear thermoelasticity have been contributed to the field (see $[14,15,17,18,26,48,49]$ and references therein).

The focus of this paper is on thermoelastic plates and associated uniform stability issues. This particular class of problems has received considerable attention

The research of I. Lasiecka has been partially supported by DMS-NSF Grant Nr 0606882.

S. Maad was supported by the Swedish Research Council and by the European Union under the Marie Curie Fellowship MEIF-CT-2005-024191. 
in recent years, particularly in the the context of some new developments in control theory. Questions such as exponential stability, controllability, observability, unique continuation have been asked and partially answered for both linear and nonlinear plates (see [30] and references therein). This includes discoveries such as

(1) exponential decays (without any mechanical dissipation) of energy in linear models $[4,5,11,27]$,

(2) boundary controllability and null controllability of linear plates [3, 6, 7, 12, 19, $22,23,29,35,39]$,

(3) analyticity of semigroups generated by linear models [37, 40, 43],

(4) unique continuation from the boundary and backward uniqueness $[20,28,38]$, and

(5) well-posedness and uniform decays of energy in semilinear thermoelastic models $[8,26,32-34]$.

While there is at present vast literature dealing with well-posedness and stability of linear and semilinear thermoelastic equations (see above), the treatment of quasilinear and fully nonlinear models defined on multidimensional domains is much more subtle and requires different mathematical approaches.

A distinct feature of this paper is that it deals with global solutions, without any smallness assumptions, to a multi-dimensional quasilinear thermoelastic plate model. One of the fundamental difficulties is that perturbation type or fixed point type of arguments, quite successful in semilinear analysis, are no longer applicable to the strongly nonlinear cases. A different approach that is capable of handling nonlinear terms in the equation is called for. It turns out that rather recent sharp estimates, developed in the context of linear control theory, allows successful handling of quasilinear models. This is the case in dealing with issues such as passing a weak limit on nonlinear terms, accomplished by taking advantage of compensated compactness methods based on nonlocal (PDO) multipliers, in deriving inverse type inequalities. The latter is the necessary ingredient for stabilization, which depends on recently developed observability estimates for thermoelastic plates [4,13].

The equations we consider arise from a model that takes into account the coupling between elastic, magnetic and thermal fields in a nonlinear elastic plate model (see $[2,9,24,25,41])$. In non-dimensional form, the equations we consider are given below in (1.1)-(1.3). Although we consider the case when $n \leq 3$, similar equations were derived when $n \leq 2$ for a current carrying plate in a magnetic field, with the consideration of a physical nonlinearity of the plate material (see $[2,24$, $25]$ ). The nonlinearity arises from the nature of the magnetoelastic material, owing to a nonlinear dependence between the intensities of the deformation and stress. We also assume that the material nonlinearity is cubic, as in the original plate model [24].

Let $\Omega$ be a bounded domain of $\mathbb{R}^{n}, n \leq 3$, with a smooth boundary $\partial \Omega$. Consider the system

$$
\left\{\begin{array}{r}
W_{t t}+\Delta^{2} W-\Delta \Theta+a \Delta\left((\Delta W)^{3}\right)=0 \\
\Theta_{t}-\Delta \Theta+\Delta W_{t}=0
\end{array}\right\} \text { in } \Omega \times(0, T)
$$




$$
\begin{aligned}
& W=\Delta W=\Theta=0 \text { on } \partial \Omega \times(0, T) \quad \text { (Boundary Conditions) } \\
& \left\{\begin{array}{lll}
W(x, 0)=f(x) & (x \in \Omega) ; & f \in W^{2,4}(\Omega) \cap W_{0}^{1,2}(\Omega) \\
W_{t}(x, 0)=g(x) & (x \in \Omega) ; & g \in L^{2}(\Omega) \\
\Theta(x, 0)=h(x) & (x \in \Omega) ; & h \in L^{2}(\Omega)
\end{array}\right\} \quad \text { (Initial Conditions). }
\end{aligned}
$$

We assume that the material constant $a$ is positive.

In this paper we study global existence and uniform decays in time of solutions $\left(W, W_{t}, \Theta\right) \in L^{\infty}\left([0, T] ; W^{2,4}(\Omega) \times L^{2}(\Omega) \times L^{2}(\Omega)\right)$ to the above initial/boundary value problem, where $T>0$ is arbitrary.

In order to proceed with the exposition of our results, we introduce some notation and definitions. Let

$$
\begin{aligned}
& X:=\left(W^{2,4}(\Omega) \cap W_{0}^{1,2}(\Omega)\right) \times L^{2}(\Omega) \times L^{2}(\Omega) \\
& Y:=L^{2}(\Omega) \times\left(W^{2,4}(\Omega) \cap W_{0}^{1,2}(\Omega)\right)^{\prime} \times\left(W^{2,2}(\Omega)\right)^{\prime} .
\end{aligned}
$$

Definition 1.1 (Weak solution). Let $0<T \leq \infty$. By a weak solution of the initial/boundary value problem (1.1)-(1.3) we mean a triple

$$
x:=(W, U, \Theta) \in L^{\infty}([0, T) ; X) \cap W^{1, \infty}([0, T) ; Y), \quad \text { with } \quad \Theta \in L^{2}\left([0, T) ; W_{0}^{1,2}(\Omega)\right)
$$

such that the following equalities hold for almost all $t \in[0, T)$ and all test functions $\varphi_{1} \in L^{2}(\Omega), \varphi_{2} \in W^{2,4}(\Omega) \cap W_{0}^{1,2}(\Omega), \varphi_{3} \in W^{2,2}(\Omega) \cap W_{0}^{1,2}(\Omega)$ :

$$
\begin{aligned}
\left\langle W_{t}, \varphi_{1}\right\rangle & =\left\langle U, \varphi_{1}\right\rangle \\
\left\langle U_{t}, \varphi_{2}\right\rangle & =-\left\langle\Delta W, \Delta \varphi_{2}\right\rangle-\left\langle\nabla \Theta, \nabla \varphi_{2}\right\rangle-a\left\langle(\Delta W)^{3}, \Delta \varphi_{2}\right\rangle \\
\left\langle\Theta_{t}, \varphi_{3}\right\rangle & =-\left\langle\nabla \Theta, \nabla \varphi_{3}\right\rangle-\left\langle U, \Delta \varphi_{3}\right\rangle,
\end{aligned}
$$

where $\langle\cdot, \cdot\rangle$ denotes the inner product in $L^{2}(\Omega)$ as well as the pairing of $L^{4}(\Omega)$ with its dual space $L^{4 / 3}(\Omega)$.

In addition, the initial conditions $(1.3)$ are satisfied in the $C_{w}([0, T) ; X)$ topology, where $C_{w}([0, T) ; X)$ denotes the space of weakly continuous functions with values in $X$

Remark 1.2. Note that with $(W, U, \Theta) \in L^{\infty}([0, T] ; X)$ and $\left(W_{t}, U_{t}, \Theta_{t}\right) \in$ $L^{\infty}([0, T] ; Y)$, we actually have

$$
(W, U, \Theta) \in C\left([0, T] ; W^{1,2}(\Omega) \times\left(W^{2,4}(\Omega)\right)^{\prime} \times\left(W^{2,2}(\Omega)\right)^{\prime}\right),
$$

see $[21$, p. 286-289]. In particular, $x=(W, U, \Theta)$ is weakly continuous with respect to the above extended topologies. By Lemma 3.3 in [52], $x$ is weakly continuous with the values in $X$. Moreover, $x \in C([0, T] ; Y)$ where $X \subset Y$ with compact injection. In view of the above, the initial conditions can be interpreted either via weak continuity with values in $X$ or via strong continuity with values in $X_{1}=$ $W^{2-\epsilon, 4}(\Omega) \times H^{-\epsilon}(\Omega) \times H^{-\epsilon}(\Omega)$, for every $\epsilon>0$. 
It is not difficult to show that every classical solution $(W, \Theta)$ of the initial/boundary value problem (1.1)-(1.3) gives a weak solution $\left(W, W_{t}, \Theta\right)$ to the system (1.4)-(1.6), and conversely (see Section 2.6) that every weak solution $(W, U, \Theta)$ of $(1.4)-(1.6)$ which is sufficiently smooth satisfies $W_{t}=U$ and $(W, \Theta)$ is a classical solution to the system (1.1)-(1.3). By forcing $W$ and $\Theta$ to be in $W_{0}^{1,2}(\Omega)$ for a.e. $t \in[0, T]$, we ensure that the boundary conditions $\left.W\right|_{\partial \Omega}=0$ and $\left.\Theta\right|_{\partial \Omega}=0$ are satisfied. The remaining boundary condition $\left.\Delta W\right|_{\partial \Omega}=0$ appears as a natural boundary condition and will follow from the weak formulation of the system (see Section 2.6).

For all $(w, u, \theta) \in X$ we define the energy of the system $\mathcal{E}: X \rightarrow \mathbb{R}$ given by

$$
\mathcal{E}(w, u, \theta)=\frac{1}{2}\|u\|_{L^{2}}^{2}+\frac{1}{2}\|\Delta w\|_{L^{2}}^{2}+\frac{1}{2}\|\theta\|_{L^{2}}^{2}+\frac{a}{4}\|\Delta w\|_{L^{4}}^{4} .
$$

If $x=(W, U, \Theta) \in L^{\infty}([0, T) ; X)$ is a weak solution, then we also define the energy (corresponding to $x$ ) by

$$
\begin{aligned}
E(t):=\mathcal{E}(W(t), U(t), \Theta(t))= & \frac{1}{2}\|U(t)\|_{L^{2}}^{2} \\
& +\frac{1}{2}\|\Delta W(t)\|_{L^{2}}^{2}+\frac{1}{2}\|\Theta(t)\|_{L^{2}}^{2}+\frac{a}{4}\|\Delta W(t)\|_{L^{4}}^{4} .
\end{aligned}
$$

Thus

$$
E(0)=\frac{1}{2}\|g\|_{L^{2}}^{2}+\frac{1}{2}\|\Delta f\|_{L^{2}}^{2}+\frac{1}{2}\|h\|_{L^{2}}^{2}+\frac{a}{4}\|\Delta f\|_{L^{4}}^{4} .
$$

The main result pertaining to global existence of finite energy solutions is the following:

Theorem 1.3 (Global existence of finite energy solutions). Let $0<T \leq \infty$ and $n \leq 3$. Then there exists a weak solution (in the sense of Definition 1.1) of the initial/boundary value problem (1.1)-(1.3). Moreover, the energy inequality

$$
E(t)+\int_{s}^{t}\|\nabla \Theta(s)\|_{L^{2}}^{2} d s \leq E(s), \quad s<t
$$

holds for this weak solution.

Remark 1.4. The global solutions described by Theorem 1.3 are obtained via the Faedo-Gelerkin method. It is shown that finite dimensional Galerkin approximations converge strongly to weak solutions. In view of the above, the proof of Theorem 1.3 also provides an effective strongly convergent finite dimensiuonal approximation of weak solutions.

Once global existence of finite energy solutions is established, a natural question to ask is that of asymptotic stability. The dissipative mechanism in the model is exhibited by the thermal component of the system. The nonlinear mechanical component has no dissipation whatsoever and does not - by itself - cause any decrease of the energy. Thus any hope for having uniform decays of the energy must be based on a possibility of propagating the decay from the thermal component of the system (heat equation) onto the mechanical component (plate equation). 
And in fact this is indeed the case in linear models, where exponential decay rates for the linear energy have been established $[4,5,27]$ for linear thermoelastic plates and more recently in $[8,13]$ for semilinear plates. The situation in the quasilinear case is much more complex, due to the unboundedness of the nonlinear term with respect to the topology induced by the energy. Nevertheless, we will be able to show that for the initial conditions taken from any ball $B_{X}(0, R)$ in $X$, the corresponding weak solution decays exponentially to zero with a rate depending on $R$ (of arbitrary size) only (and not on the particular solution). The corresponding result is the following:

Theorem 1.5 (Exponential decay of the energy). Let $n \leq 3, T=\infty$, and $R>0$. Then there exists a constant $C$ (independent of $R$ ) and a constant $\omega_{R}$ (depending on $R$ ) such that if $(W(t), U(t), \Theta(t))$ is a solution of the initial/boundary value problem (1.1)-(1.3) obtained in Theorem 1.3, with $E(0) \leq R$, then

$$
\text { for all } t \geq 0, \quad E(t) \leq C E(0) e^{-\omega_{R} t} \text {. }
$$

Note that the constant $\omega$ given in (1.8) in Theoorem 1.5 depends on the size of initial data $E(0) \leq R$ (this dependence is made explicit in the course of the proof of the theorem). One may give a different formulation for the decay rates, formula with the decay rate constant $\omega$ independent on $R$. The price to pay is the fact that the amplitude constant $C$ will depend on $R$. The corresponding result is formulated below.

Corollary 1.6. Under the assumptions of Theorem 1.5 and with $E(0) \leq R$ we have:

$$
\text { for all } t \geq 0, \quad E(t) \leq C(R, \omega) e^{-\omega t} .
$$

where $\omega$ is any positive number such that $\omega<\omega_{0} \equiv \frac{1}{T_{0}} \log \frac{T_{0}+\lambda_{1}^{-1}}{7 \lambda_{1}^{-1}}, T_{0}$ is a solution of the transcendental equation (3.18) and $\lambda_{1}$ first eigenvalue of $-\Delta$ equipped with Dirichlet boundary conditions. The $R \mapsto C(R, \omega)$ is increasing and $C(R, \omega) \rightarrow \infty$ as $\omega \rightarrow \omega_{0}$.

Remark 1.7. The constant $\omega_{R}$ appearing in (1.8) becomes small when $R$ is large. (The explicit dependence is given in the course of the proof.) This leads to slow decay rates for large initial data. Instead, the constant $\omega$ in (1.9) is independent on $R$. Thus, in the case of large initial data the estimate given by (1.9) leads to much faster decay rates.

Remark 1.8. The decay rates given by (1.8) or (1.9) apply to weak solutions that are obtained in Theorem 1.3 as the strong limits of Faedo-Galerkin approximations. Since weak solutions, as defined by Definition 1.1, may not be unique, we can not extend the validity of (1.8) to all weak solutions. However, the decay rates given in (1.8) remain valid for all solutions that are sufficiently regular.

Remark 1.9. The two theorems stated above pertain to existence and uniform decays of weak, or alternatively, finite energy solutions. With further restrictions imposed on the initial data, one can prove existence and uniqueness of regular 
(in fact, classical) solutions. The corresponding result, which relies on methods of nonlinear analytic semigroups and maximal regularity [44], will be given in the Appendix.

\section{Existence of a solution}

In this section, we prove Theorem 1.3.

We note that the system represented by (1.1)-(1.3) can be seen as a nonlinear perturbation of an analytic semigroup (see the Appendix). However, the resulting perturbation is not relatively bounded with respect to the generator, and so perturbation theory for analytic semigroups [47] cannot be applied. This presents major difficulty in studying existence of the finite energy solution claimed by Theorem 1.3. In order to handle the difficulty, we shall resort to the compensated compactness method along with the use of partial monotonicity generated by the nonlinear term (the problem itself is not monotone!). The latter property is instrumental in identifying limits correctly in the weak formulation.

\subsection{Lyapunov function for the PDE system}

Suppose that the system (1.4)-(1.6) has a solution

$(W, U, \Theta) \in C^{1}\left([0, T] ;\left(W^{2,4}(\Omega) \cap W_{0}^{1,2}(\Omega)\right) \times W^{1,2}(\Omega) \times\left(W^{2,2}(\Omega) \cap W_{0}^{1,2}(\Omega)\right)\right)$.

It follows that $\left(W_{t}, U_{t}, \Theta_{t}\right) \in C\left([0, T] ;\left(W^{2,4}(\Omega) \cap W_{0}^{1,2}(\Omega)\right) \times W^{1,2}(\Omega) \times\left(W^{2,2}(\Omega) \cap\right.\right.$ $\left.\left.W_{0}^{1,2}(\Omega)\right)\right)$. Let

$$
E(t)=\frac{1}{2}\|U(t)\|_{L^{2}}^{2}+\frac{1}{2}\|\Delta W(t)\|_{L^{2}}^{2}+\frac{1}{2}\|\Theta(t)\|_{L^{2}}^{2}+\frac{a}{4}\|\Delta W(t)\|_{L^{4}}^{4} .
$$

Then by (1.4)-(1.6),

$$
\begin{aligned}
\frac{d}{d t} E(t) & =\left\langle U, U_{t}\right\rangle+\left\langle\Delta W, \Delta W_{t}\right\rangle+\left\langle\Theta, \Theta_{t}\right\rangle+a\left\langle(\Delta W)^{3}, \Delta W_{t}\right\rangle \\
& =\left\langle\Theta, \Delta W_{t}\right\rangle+\left\langle\Theta, \Theta_{t}\right\rangle \\
& =\langle\Theta, \Delta \Theta\rangle=-\|\nabla \Theta\|_{L^{2}}^{2} \leq 0 .
\end{aligned}
$$

The above argument is only formal, since it relies on additional regularity of the solutions. On the other hand, it indicates that the sought after solutions should have a-priori bounds in the topology of $X$ and that the energy is non-increasing, suggesting some sort of dissipation. We shall make this argument rigorous by considering the appropriate finite dimensional approximations of the original system. Weak lower semicontinuity of the energy functional will allow us to conclude that the energy inequality is valid for the original PDE system. We also note that the dissipation is weak, since it affects only one component of the state vector. Nevertheless we will be able to show that this effect propagates, giving exponential decay rates on the entire system (see Theorem 1.5). 


\subsection{Faedo-Galerkin approximations}

Let $\left(e_{k}\right)_{k \in \mathbb{N}}$ be normalized eigenfunctions of the negative Laplacian with Dirichlet boundary conditions:

$$
\begin{aligned}
-\Delta e_{k} & =\lambda_{k} e_{k} \quad \text { in } \quad \Omega, \\
\left.e_{k}\right|_{\partial \Omega} & =0 .
\end{aligned}
$$

The $\left\{e_{k} \mid k \in \mathbb{N}\right\}$ is an orthogonal basis of $W_{0}^{1,2}(\Omega)$, and form an orthonormal basis of $L^{2}(\Omega)$. Let $V^{N}:=\operatorname{span}\left\{e_{m} \mid m=1, \ldots, N\right\}$ and $X^{N}:=V^{N} \times V^{N} \times V^{N}$.

We seek

$$
\begin{aligned}
W_{N}(x, t) & =\sum_{k=1}^{N} w_{k}^{N}(t) e_{k}(x) \\
U_{N}(x, t) & =\sum_{k=1}^{N} u_{k}^{N}(t) e_{k}(x) ; \quad \Theta_{N}(x, t)=\sum_{k=1}^{N} \theta_{k}^{N}(t) e_{k}(x)
\end{aligned}
$$

which satisfy

$$
\begin{aligned}
& \left\{\begin{array}{r}
\left\langle U^{N}-W_{t}^{N}, e_{m}\right\rangle=0 \\
\left\langle U_{t}^{N}+\Delta^{2} W^{N}-\Delta \Theta^{N}+a \Delta\left(\left(\Delta W^{N}\right)^{3}\right), e_{m}\right\rangle=0 \\
\left\langle\Theta_{t}^{N}-\Delta \Theta^{N}+\Delta U^{N}, e_{m}\right\rangle=0
\end{array}\right\} \quad m \in\{1, \ldots, N\} \\
& \left\{\begin{array}{r}
W^{N}(x, 0)=\sum_{k=1}^{N}\left\langle f, e_{k}\right\rangle e_{k}(x) \\
U^{N}(x, 0)=\sum_{k=1}^{N}\left\langle g, e_{k}\right\rangle e_{n}(x) \\
\Theta^{N}(x, 0)=\sum_{k=1}^{N}\left\langle h, e_{k}\right\rangle e_{k}(x)
\end{array}\right\} \text { on } \Omega \quad \text { (Initial Conditions). }
\end{aligned}
$$

\subsection{System of ODEs}

We note that $\left(W^{N}, U^{N}, \Theta^{N}\right)$ given by (2.1) satisfy $(2.2)-(2.3)$ if and only if the coefficient functions $\left(w_{1}^{N}, \ldots, w_{N}^{N}, u_{1}^{N}, \ldots, u_{N}^{N}, \theta_{1}^{N}, \ldots, \theta_{N}^{N}\right)$ satisfy the following system of ODEs:

$$
\begin{aligned}
& \left\{\begin{array}{l}
\dot{w}_{m}^{N}(t)=u_{m}^{N}(t) \\
\dot{u}_{m}^{N}(t)=-\lambda_{m}^{2} w_{m}^{N}(t)+\lambda_{m} \theta_{m}^{N}(t)+a\left\langle\left(\sum_{k=1}^{N} w_{k}^{N}(t) \Delta e_{k}\right)^{3}, \lambda_{m} e_{m}\right\rangle \\
\dot{\theta}_{m}^{N}(t)=-\lambda_{m} \theta_{m}^{N}(t)-\lambda_{m} u_{m}^{N}(t)
\end{array}\right\} \\
& m \in\{1, \ldots, N\}
\end{aligned}
$$

with the initial conditions

$$
\left\{\begin{array}{r}
w_{m}^{N}(0)=\left\langle f, e_{m}\right\rangle \\
u_{m}^{N}(0)=\left\langle g, e_{m}\right\rangle \\
\theta_{m}^{N}(0)=\left\langle h, e_{n}\right\rangle
\end{array}\right\} \quad m \in\{1, \ldots, N\} .
$$

As the right hand side of (2.4) is locally Lipschitz, it follows that the above system of ODEs has a local solution in a maximal time interval $\left[0, T_{N}\right)$ for some $T_{N}>0$. Multiplying (2.4) by $e_{m}$ and adding the results, we obtain (2.2)-(2.3). 


\subsection{Lyapunov function for the ODE system}

Let $x^{N}:=\left(W^{N}, U^{N}, \Theta^{N}\right)$, and define $\mathcal{E}_{N}: \mathbb{R}^{3 N} \rightarrow \mathbb{R}$ by

$$
\begin{aligned}
\mathcal{E}_{N}\left(x^{N}\right):= & \mathcal{E}\left(x^{N}\right) \\
= & \frac{1}{2}\left(\left\|\Delta W^{N}\right\|_{L^{2}}^{2}+\left\|U^{N}\right\|_{L^{2}}^{2}+\left\|\Theta^{N}\right\|_{L^{2}}^{2}\right)+\frac{1}{4}\left\|\Delta W^{N}\right\|_{L^{4}}^{4} \\
= & \frac{1}{2}\left\|\sum_{k=1}^{N} u_{k} e_{k}\right\|_{L^{2}}^{2}+\frac{1}{2}\left\|\sum_{k=1}^{N} \lambda_{k} w_{k} e_{k}\right\|_{L^{2}}^{2}+\frac{1}{2}\left\|\sum_{k=1}^{N} \theta_{k} e_{k}\right\|_{L^{2}}^{2} \\
& +\frac{a}{4}\left\|\sum_{k=1}^{N} \lambda_{k} w_{k} e_{k}\right\|_{L^{4}}^{4} .
\end{aligned}
$$

It follows from the linear independence of $e_{k}$ 's and $\Delta e_{k}$ 's that $\mathcal{E}_{N}$ is positive definite.

$$
\begin{aligned}
& \text { If }\left(W^{N}, U^{N}, \Theta^{N}\right) \text { given by (2.1) satisfy }(2.2)-(2.3) \text {, then we define } \\
& E_{N}(t):=\mathcal{E}_{N}\left(w_{1}^{N}(t), \ldots, w_{N}^{N}(t), u_{1}^{N}(t), \ldots, u_{N}^{N}(t), \theta_{1}^{N}(t), \ldots, \theta_{N}^{N}(t)\right) .
\end{aligned}
$$

It can be verified that

$$
\frac{d}{d t} E_{N}(t)=-\left\|\sum_{k=1}^{N} \theta_{k}^{N}(t) \nabla e_{k}\right\|_{L^{2}}^{2}=-\left\|\nabla \Theta^{N}\right\|_{L^{2}} \leq 0 .
$$

So we can conclude that the solution to (2.4) with initial conditions (2.5) is bounded. Consequently from ODE theory, we obtain existence and uniqueness of the finite-dimensional solution $x^{N} \in C\left(\left[0, T_{N}\right] ; X^{N}\right)$ satisfying the ODE system, which in addition, is a-priori bounded. Thus $T_{N}=+\infty$.

\subsection{Uniform bounds}

Since

$$
\frac{d}{d t} E_{N}(t)=-\left\|\nabla \Theta^{N}(t)\right\|_{L^{2}}^{2} \leq 0,
$$

it follows that $E_{N}(t) \leq E_{N}(0)$. But

$$
E_{N}(0)=\frac{1}{2}\left\|U_{N}(0)\right\|_{L^{2}}^{2}+\frac{1}{2}\left\|\Delta W_{N}(0)\right\|_{L^{2}}^{2}+\frac{1}{2}\left\|\Theta_{N}(0)\right\|_{L^{2}}^{2}+\frac{a}{4}\left\|\Delta W_{N}\right\|_{L^{4}}^{4} .
$$

Consequently

$$
E_{N}(0) \leq \frac{1}{2}\|g\|_{L^{2}}^{2}+\frac{1}{2}\|\Delta f\|_{L^{2}}^{2}+\frac{1}{2}\|h\|_{L^{2}}^{2}+\frac{a}{4}\|\Delta f\|_{L^{4}}^{4}=E(0) .
$$

In particular, $E_{N}(0)$ is uniformly bounded in $N$. 


\subsection{Weak solution}

In order to show that the definition of weak solution is meaningful and describes the original PDE problem, we need to verify that every classical solution is a weak solution, and that every weak solution which is sufficiently smooth is a classical solution. The first part is straightforward and it follows by projecting the classical solution on the $L^{2}$ space. For the second part, consider a weak solution that is sufficiently smooth. In the formulation of weak solutions, we first take test functions $\phi_{i}$ which are in $C_{0}^{\infty}(\Omega)$. It is easy to show by a straightforward application of Green's formula and the density of $C_{0}^{\infty}(\Omega)$ in $L^{2}(\Omega)$, that the equations (1.1) are satisfied. It suffices to reconstruct the boundary condition $\Delta W=0$ on $\partial \Omega$. (The other two boundary conditions are encoded in the definition of the weak solution.) By using (1.1) in the weak formulation, applied with an arbitrary test function $\phi_{2} \in W^{2,4}(\Omega) \cap W_{0}^{1.2}(\Omega)$, and integrating by parts, we obtain the following trace relations:

$$
\int_{\partial \Omega}\left(\Delta W-\Theta+a(\Delta W)^{3}\right) \frac{\partial}{\partial \nu} \phi_{2} d s=0
$$

for all $\phi_{2} \in W^{2,4}(\Omega) \cap W_{0}^{1.2}(\Omega)$. By the surjectivity in the trace theorem, it follows that for any $z \in W^{3 / 4,4}(\partial \Omega)$, there exists a $\phi_{2} \in W^{2,4}(\Omega) \cap W_{0}^{1.2}(\Omega)$ such that $\frac{\partial}{\partial \nu} \phi_{2}=z$ on $\partial \Omega[53]$. Hence

$$
\int_{\partial \Omega}\left(\Delta W-\Theta+a(\Delta W)^{3}\right) z d s=0
$$

for all $z \in W^{3 / 4,4}(\partial \Omega)$. Since $\Theta=0$ on $\partial \Omega$, it follows by density that

$$
\Delta W+a(\Delta W)^{3}=0 \quad \text { on } \quad \partial \Omega .
$$

Consequently, $\Delta W=0$ on $\partial \Omega$ (since $a>0$ ), as desired.

\subsection{Weak convergence of the Faedo-Galerkin approximations}

From the uniform Lyapunov estimate (2.9) for the Galerkin approximations, we obtain that for all $t \in[0, T]$,

$$
\left\|\Delta W_{N}(\cdot, t)\right\|_{L^{2}}, \quad\left\|\Delta W_{N}(\cdot, t)\right\|_{L^{4}}, \quad\left\|U_{N}(\cdot, t)\right\|_{L^{2}}, \quad\left\|\Theta_{N}(\cdot, t)\right\|_{L^{2}}
$$

are all bounded sequences. Also $\int_{0}^{T}\left\|\nabla \Theta^{N}(t)\right\|_{L^{2}} d t$ is bounded by the norms of the initial data. Moreover, after accounting for $W_{N}=\Theta_{N}=0$ on the boundary, elliptic theory and the Poincaré inequality give control of the $W^{2,4}(\Omega)$ norm of $W_{N}$ and the $H_{0}^{1}(\Omega)$ norm of $\Theta_{N}$. Thus

$$
\begin{array}{lll}
W_{N} \text { is a bounded sequence in } & L^{\infty}\left([0, T] ; W^{2,4}(\Omega)\right), \\
U_{N} \text { is a bounded sequence in } & L^{\infty}\left([0, T] ; L^{2}(\Omega)\right), \\
\Theta_{N} \text { is a bounded sequence in } & L^{\infty}\left([0, T] ; L^{2}(\Omega)\right) \cap L^{2}\left([0, T] ; H_{0}^{1}(\Omega)\right) .
\end{array}
$$


Step 1. Weak convergence. Therefore there exist subsequences which converge in the weak star topology of the respective spaces. We denote the respective limits by $W, U, \Theta$. For simplicity of notation, we renumber the sequences and assume without loss of generality that the sequences themselves converge in the weak star topology of the appropriate spaces.

Thus

$$
\begin{aligned}
& W^{N} \rightarrow^{*} W \quad \text { in } \quad L^{\infty}\left([0, T] ; W^{2,4}(\Omega)\right), \\
& U^{N} \rightarrow^{*} U \text { in } \quad L^{\infty}\left([0, T] ; L^{2}(\Omega)\right), \\
& \Theta^{N} \rightarrow^{*} \Theta \quad \text { in } \quad L^{\infty}\left([0, T] ; L^{2}(\Omega)\right), \\
& \Theta^{N} \rightarrow \Theta \quad \text { in } \quad L^{2}\left([0, T] ; H^{1}(\Omega)\right),
\end{aligned}
$$

where we have also used the reflexivity of $L^{2}\left([0, T] ; H^{1}(\Omega)\right)$. We also obtain that

$$
\begin{gathered}
U_{t}^{N} \rightarrow U_{t} \quad \text { in } \quad L^{2}\left([0, T] ; W^{2,4}(\Omega)^{\prime}\right), \\
\Delta^{-1} \Theta_{t}^{N} \rightarrow^{*} \Delta^{-1} \Theta_{t} \quad \text { in } \quad L^{\infty}\left([0, T] ; L^{2}(\Omega)\right),
\end{gathered}
$$

where $\Delta^{-1}$ denotes the inverse of the Laplacian with zero Dirichlet boundary conditions. In particular, by the Aubin-Simon Lemma [51], this implies that:

$$
\begin{array}{cccc}
W_{N} \rightarrow W & \text { strongly in } & C\left([0, T] ; H^{2-\epsilon}(\Omega)\right), \\
\Theta_{N} \rightarrow \Theta & \text { strongly in } & C\left([0, T] ; H^{-\epsilon}(\Omega)\right), \\
\Theta_{N} \rightarrow \Theta & \text { strongly in } & L^{2}\left([0, T] ; H^{1-\epsilon}(\Omega)\right),
\end{array}
$$

where $\epsilon>0$ can be taken arbitrarily small.

The above convergence allows us to pass the limit on all linear terms in the weak formulation of the system. The passage of the limit on the nonlinear term is more involved and requires additional arguments. Indeed, note that since $\Delta W^{N}(t)$ is uniformly bounded in $L^{4}(\Omega)$, it follows that $\left(\Delta W^{N}\right)^{3}$ is bounded in $L^{\infty}\left([0, T] ; L^{4 / 3}(\Omega)\right)$. So there exists an $\eta \in L^{\infty}\left([0, T] ; L^{4 / 3}(\Omega)\right)$ such that $\left(\Delta W^{N}\right)^{3}$ $\rightarrow^{*} \eta$ in $L^{\infty}\left([0, T] ; L^{4 / 3}(\Omega)\right)$ (again on a subsequence). But we do not know if $\eta$ coincides with $(\Delta W)^{3}$, since we do not have any compactness to conclude this. As we shall see later, this desired conclusion will be drawn by exhibiting some sort of compensated compactness.

Let $\varphi \in C_{0}^{\infty}([0, T] ; \mathbb{R})$ and $m \in \mathbb{N}$ be arbitrary. From $(2.2)$ we have

$$
\begin{array}{r}
\int_{0}^{T}\left(\left\langle U_{t}^{N}, e_{m}\right\rangle+\left\langle\Delta W^{N}-\Theta^{N}+a\left(\left(\Delta W^{N}\right)^{3}\right), \Delta e_{m}\right\rangle\right) \varphi(t) d t=0 \\
\int_{0}^{T}\left(\left\langle\Theta_{t}^{N}, e_{m}\right\rangle-\left\langle\Theta^{N}+U^{N}, \Delta e_{m}\right\rangle\right) \varphi(t) d t=0
\end{array}
$$


for $N \geq m$. Letting $N \rightarrow \infty$ and using the weak star convergence of $W^{N}, U^{N}$, $U_{t}^{N}, \Theta^{N}, \Theta_{t}^{N}$ and $\left(\Delta W^{N}\right)^{3}$, we conclude that

$$
\begin{aligned}
\int_{0}^{T}\left(\left\langle U_{t}, e_{m}\right\rangle+\left\langle\Delta W-\Theta+a \eta, \Delta e_{m}\right\rangle\right) \varphi(t) d t & =0 \\
\int_{0}^{T}\left(\left\langle\Theta_{t}, e_{m}\right\rangle-\left\langle\Theta+U, \Delta e_{m}\right\rangle\right) \varphi(t) d t & =0 .
\end{aligned}
$$

Since $\varphi \in C_{0}^{\infty}([0, T] ; \mathbb{R})$ was arbitrary and any test function $\psi \in W^{2,4}(\Omega)$ can be approximated (with strong convergence) by finite linear combinations of $e_{m}$, it follows that

$$
\begin{aligned}
\left\langle U_{t}, \psi\right\rangle+\langle\Delta W-\Theta+a \eta, \Delta \psi\rangle & =0 \\
\left\langle\Theta_{t}, \psi\right\rangle-\langle\Theta+U, \Delta \psi\rangle & =0
\end{aligned}
$$

for almost every $t \in[0, T]$. Because $W_{0}^{1,2}(\Omega)$ is a closed subspace of $W^{1,2}(\Omega)$, and hence weakly closed, it follows that also $W(t) \in W_{0}^{1,2}(\Omega)$.

Next we show that the initial conditions are satisfied. Let

$$
\varphi \in L^{1}\left([0, T] ; W^{2,4}(\Omega) \cap W_{0}^{1,2}(\Omega)\right) \cap C^{\infty}\left([0, T] ; C^{\infty}(\Omega)\right)
$$

be such that $\varphi(T)=0$. Then

$$
\begin{aligned}
\int_{0}^{T}\left\langle U^{N}, \varphi\right\rangle d t & =\int_{0}^{T}\left\langle W_{t}^{N}, \varphi\right\rangle d t=-\left\langle f^{N}, \varphi(0)\right\rangle-\int_{0}^{T}\left\langle W^{N}, \varphi_{t}\right\rangle d t \\
\int_{0}^{T}\langle U, \varphi\rangle d t & =\int_{0}^{T}\left\langle W_{t}, \varphi\right\rangle d t=-\langle W(0), \varphi(0)\rangle-\int_{0}^{T}\left\langle W, \varphi_{t}\right\rangle d t .
\end{aligned}
$$

By the weak star convergence, we obtain $f^{N} \rightarrow W(0)$. From the construction, we also have $f^{N} \rightarrow f$, and so $\langle W(0), \varphi(0)\rangle=\langle f, \varphi(0)\rangle$. Since $\varphi$ was arbitrary, it follows that $W(0)=f$. In the same way, one shows that $U(0)=g$ and that $\Theta(0)=h$.

Step 2. Strong convergence of the velocity. The main task that remains is to identify $\eta$ with $(\Delta W)^{3}$. The proof of this fact will proceed through several lemmas. To achieve this, we first improve the convergence of $U^{N}$.

Lemma 2.1. Let $n \leq 3$. Then $U^{N} \rightarrow U$ strongly in $L^{2}\left([0, T] ; L^{2}(\Omega)\right)$.

Proof. Let $\left(W^{N}, U^{N}, \Theta^{N}\right)$ denote the Galerkin approximation at step $N$, and let $(W, U, \Theta)$ be its weak limit asserted in $(2.10)$. We shall use the notation $\widetilde{W}^{N}:=$ $W^{N}-W$, and a similar notation is used for the other two variables. Let $P_{N}$ be the orthogonal projection (with respect to the inner product of $L^{2}(\Omega)$ ) of the space $V:=W^{2,4}(\Omega) \cap H_{0}^{1}(\Omega)$ onto $V^{N}$.

We obtain the following system satisfied for $\widetilde{W}^{N}, \widetilde{\Theta}^{N}$ and all test functions $\psi, \phi \in V:$

$$
\begin{aligned}
\left\langle\widetilde{U}_{t}^{N}+\Delta^{2} \widetilde{W}^{N}-\Delta \widetilde{\Theta}^{N}+a \Delta\left(\left(\Delta W^{N}\right)^{3}-\eta\right), \psi\right\rangle & =\left\langle\beta_{N},\left(I-P_{N}\right) \psi\right\rangle \\
\left\langle\widetilde{\Theta}_{t}^{N}-\Delta \widetilde{\Theta}^{N}+\Delta \widetilde{U}^{N}, \phi\right\rangle & =\left\langle\gamma_{N},\left(I-P_{N}\right) \phi\right\rangle,
\end{aligned}
$$


where the error terms are

$$
\beta_{N}:=U_{t}^{N}+\Delta^{2} W^{N}-\Delta \Theta^{N}+a \Delta\left(\Delta W^{N}\right)^{3} \quad \text { and } \quad \gamma_{N}:=\Theta_{t}^{N}-\Delta \Theta^{N}+\Delta U^{N} \text {. }
$$

We will derive the error for $\widetilde{U}^{N}$ by using suitable multipliers. An important fact which will be used without further mentioning is that both $\Delta$ and $\Delta^{-1}$ (with Dirichlet boundary conditions) leave $V^{N}$ invariant, and that $P_{N}$ commutes with $\Delta$. Because of this

$$
\begin{aligned}
& \left(I-P_{N}\right) \gamma_{N}=0 \\
& \left(I-P_{N}\right) \beta_{N}=a\left(I-P_{N}\right) \Delta\left(\Delta W^{N}\right)^{3} .
\end{aligned}
$$

We apply (2.14) with $\psi:=\Delta^{-1} \widetilde{\Theta}^{N}=\Delta^{-1}\left(\Theta^{N}-\Theta\right)$, and integrate from 0 to $T$. Note that $\Delta^{-1}\left(\Theta^{N}-\Theta\right)(t) \in V$ for almost all $t$. This follows from the regularity of the limit elements (2.10) and the embedding $H^{1}(\Omega) \subset L^{4}(\Omega)$ for $n \leq 3$.

Using (2.14), we obtain

$$
\begin{gathered}
\int_{0}^{T}\left(-\left\langle\widetilde{U}^{N}, \Delta^{-1} \widetilde{\Theta}_{t}^{N}\right\rangle+\left\langle\Delta \widetilde{W}^{N}, \widetilde{\Theta}^{N}\right\rangle-\left\|\widetilde{\Theta}^{N}\right\|^{2}+a\left\langle\left(\Delta W^{N}\right)^{3}-\eta, \widetilde{\Theta}^{N}\right\rangle\right) d t \\
=-\left.\left\langle\widetilde{U}^{N}, \Delta^{-1} \widetilde{\Theta}^{N}\right\rangle\right|_{0} ^{T}+\int_{0}^{T}\left\langle\beta_{N},\left(I-P_{N}\right) \Delta^{-1} \widetilde{\Theta}^{N}\right\rangle d t
\end{gathered}
$$

On the other hand, using (2.15) we also have

$$
\Delta^{-1} \widetilde{\Theta}_{t}^{N}=\widetilde{\Theta}^{N}-\widetilde{U}^{N} .
$$

Combining the last two equations, we obtain

$$
\begin{aligned}
\int_{0}^{T}\left\|\widetilde{U}^{N}\right\|_{L^{2}}^{2} d t= & \int_{0}^{T}\left(\left\langle\widetilde{U}^{N}, \widetilde{\Theta}^{N}\right\rangle-\left\langle\Delta \widetilde{W}^{N}, \widetilde{\Theta}^{N}\right\rangle\right. \\
& \left.+\left\|\widetilde{\Theta}^{N}\right\|^{2}-a\left\langle\left(\Delta W^{N}\right)^{3}-\eta, \widetilde{\Theta}^{N}\right\rangle\right) d t \\
& -\left.\left\langle\widetilde{U}^{N}(t), \Delta^{-1} \widetilde{\Theta}^{N}(t)\right\rangle\right|_{0} ^{T} \\
& +\int_{0}^{T}\left\langle\beta_{N},\left(I-P_{N}\right) \Delta^{-1} \widetilde{\Theta}^{N}\right\rangle d t .
\end{aligned}
$$

It is easy to see that all the terms on the right side of this equality converge to zero as $N \rightarrow \infty$. Indeed, uniform boundedness of $\widetilde{U}^{N}$ and $\Delta \widetilde{W}^{N}(t)$ in $L^{2}\left([0, T] ; L^{2}(\Omega)\right)$ by (2.10), along with the strong convergence $\widetilde{\Theta}^{N} \rightarrow 0$ in $L^{2}\left([0, T] ; H^{1-\epsilon}(\Omega)\right) \subset$ $L^{2}\left([0, T] ; L^{2}(\Omega)\right)$ allows us to conclude that the first three terms converge to 0 . As for the fourth term, we use uniform boundedness of $\left\|\left(\Delta \widetilde{W}^{N}\right)^{3}(t)-\eta(t)\right\|_{L^{4 / 3}}$ along with the strong convergence to zero $\widetilde{\Theta}^{N}$ in $L^{2}\left([0, T] ; H^{1-\epsilon}(\Omega)\right) \subset L^{2}\left([0, T] ; L^{4}(\Omega)\right)$ when $n \leq 3$. For the fifth term we invoke the uniform bound for $\left\|\widetilde{U}^{N}\right\|$ and strong convergence to zero of $\left\|\Delta^{-1} \widetilde{\Theta}^{N}(t)\right\|$ for all $t$. For the sixth term, we use the representation in (2.15) to obtain

$$
\left\langle\left(I-P_{N}\right) \Delta\left(\Delta W^{N}\right)^{3}, \Delta^{-1} \widetilde{\Theta}\right\rangle=\left\langle\left(\Delta W^{N}\right)^{3},\left(I-P_{N}\right) \widetilde{\Theta}\right\rangle=\left\langle\left(\Delta W^{N}\right)^{3},\left(I-P_{N}\right) \Theta\right\rangle
$$


where $\left\|\left(\Delta W^{N}(t)\right)^{3}\right\|_{L_{4 / 3}}$ is bounded and $\left(I-P_{N}\right) \Theta$ converges strongly to zero in the space $L^{2}\left([0, T] ; H^{1}(\Omega)\right)$. Finally, we conclude that $\int_{0}^{T}\left\|\widetilde{U}^{N}(t)\right\|^{2} d t \rightarrow 0$, which proves the lemma.

Step 3. Identification of the nonlinear limit. The strong convergence asserted in Lemma 2.1 allows us to prove that $\eta$ coincides with the correct quantity.

Lemma 2.2. Let $n \leq 3$. Then

$$
\left(\Delta W^{N}\right)^{3} \rightarrow(\Delta W)^{3} \quad \text { in } \quad L^{2}\left([0, T] ; L^{4 / 3}(\Omega)\right)
$$

Proof. The proof is based on a monotonicity argument and an application of [10, Lemma II.1.3]. We first note that the operator $G(W):=\Delta(\Delta W)^{3}$ is maximal monotone as considered from $W^{2,4}(\Omega)$ into its dual. We know that

$$
G\left(W^{N}\right) \rightarrow \Delta \eta \quad \text { in } \quad W^{2,4}(\Omega)^{\prime}
$$

and

$$
W^{N} \rightarrow W \quad \text { in } W^{2,4}(\Omega) .
$$

In order to identify $\eta$ with $(\Delta W)^{3}$, we invoke Lemma 3.1 in [10] which requires that

$$
\lim \sup \left\langle G\left(W_{N}\right)-\Delta \eta, W_{N}-W\right\rangle \leq 0 .
$$

In order to establish the inequality required in $(2.20)$, we go back to the first equation in (2.14). As the test function we choose $\psi:=\widetilde{W}^{N}$. Integration from 0 to $T$ gives

$$
\begin{aligned}
\int_{0}^{T}( & \left.\left\|\Delta \widetilde{W}^{N}\right\|_{L^{2}}^{2}+a\left\langle\left(\Delta W^{N}\right)^{3}-\eta, \Delta \widetilde{W}^{N}\right\rangle\right) d t \\
= & \int_{0}^{T}\left(\left\|\Delta \widetilde{W}^{N}\right\|_{L^{2}}^{2}+a\left\langle G\left(W^{N}\right)-\Delta \eta, \widetilde{W}^{N}\right\rangle\right) d t \\
= & \int_{0}^{T}\left(\left\|\widetilde{U}^{N}\right\|_{L^{2}}^{2}-\left\langle\widetilde{\Theta}^{N}, \Delta \widetilde{W}^{N}\right\rangle-\left\langle\beta_{N},\left(I-P_{N}\right) \widetilde{W}^{N}\right\rangle\right) d t \\
& -\left.\left\langle\widetilde{U}^{N}, \widetilde{W}^{N}\right\rangle\right|_{0} ^{T}
\end{aligned}
$$

We claim that all the four terms on the right hand side of (2.21) converge to zero. Indeed, the first term converges to zero by virtue of Lemma 2.1. For the second term, we invoke the uniform boundedness of $\left\|\Delta \widetilde{W}^{N}(t)\right\|_{L^{2}}$ along with strong convergence of $\Theta^{N}$ in $L^{2}\left([0, T] ; H^{1}(\Omega)\right) \subset L^{2}\left([0, T] ; L^{2}(\Omega)\right)$. For the third term we use the representation in (2.15)

$$
\left\langle\beta_{N},\left(I-P_{N}\right) \widetilde{W}\right\rangle=\left\langle\beta_{N},\left(I-P_{N}\right) W\right\rangle=\left\langle\left(\Delta W_{N}\right)^{3},\left(I-P_{N}\right) \Delta W\right\rangle \rightarrow 0,
$$

where we have used the uniform bound $\left\|\left(\Delta W_{N}\right)^{3}\right\|_{L^{4 / 3}}$ along with the strong convergence of $\left(I-P_{N}\right) \Delta W$ in $L^{4}(\Omega)$.

Finally, for the fourth term we argue as before. The bound on $\|\widetilde{U}(t)\|$ along with the strong convergence of $\widetilde{W}^{N}$ in $C\left([0, T] ; L^{2}(\Omega)\right)$ completes the argument. 
Thus, (2.20) follows from (2.21) and the fact that the right hand side of $(2.21)$ converges to 0 .

Lemma 2.2 allows us to pass the limit in the approximate equation. In addition, due to lower semicontinuity of the energy, the energy inequality holds for all weak solutions. This concludes the proof of Theorem 1.3.

Remark 2.3. Note that the result of Lemma 2.2 yields an even stronger conclusion. It says that $W^{N} \rightarrow W$ strongly in $L^{2}\left([0, T] ; W^{2,2}(\Omega)\right)$. Lemma 2.1 provides additional convergence $U^{N} \rightarrow U$ strongly in $L^{2}\left([0, T] ; L^{2}(\Omega)\right)$. This conclusion may be useful in assessing convergence of the finite-dimensional approximation to the original equation.

\section{Uniform stability of solutions}

\subsection{Exponential decay of the ODE solutions.}

From (2.8) we infer that the energy of the ODE system is nondecreasing. Since $\Theta^{N}=0$ implies that $x^{N}=0$, La Salle's invariance principle implies strong stability of the ODE system.

Our main task is to show that the obtained stability and decay rates are uniform in $N$. This will be asserted in the theorem that follows.

Theorem 3.1. Let $n \leq 3$. Then there exists a constant $C$ such that for every $R>0$ there exists a constant $\omega_{R}$ such that if $\left(W^{N}, U^{N}, \Theta^{N}\right)$ is a solution of $(2.2)-(2.3)$ with $E(0) \leq R$, then

$$
\forall t \geq 0, \quad E_{N}(t) \leq C E_{N}(0) e^{-\omega_{R} t},
$$

where $E_{N}$ is defined by (2.6) and the constants $C, \omega_{R}$ are independent on $N$.

We apply the method of multipliers introduced by Avalos and Lasiecka [5] for linear thermoelastic problems. The method uses two multipliers

$$
M_{1}(W, U, \Theta):=\Delta^{-1} \Theta \quad \text { and } \quad M_{2}(W, U, \Theta):=W,
$$

where as before $\Delta$ denotes the Laplacian with zero Dirichlet boundary conditions. We note that all the calculations are performed on finite-dimensional Galerkin solutions. This avoids potential issues with low regularity of weak solutions, where the latter may not permit for rigorous justification of PDE estimates.

If $\left(W^{N}, U^{N}, \Theta^{N}\right)$ is a solution of (2.2)-(2.3), then it follows from (2.7) that for every $t \geq 0$

$$
E_{N}(t)+\int_{0}^{t}\left\|\nabla \Theta^{N}(s)\right\|_{L^{2}}^{2} d s=E_{N}(0)
$$

We write

$$
E_{N}(t):=E_{N}^{k}(t)+E_{N}^{p}(t)
$$


where

$$
\begin{aligned}
& E_{N}^{k}(t):=\frac{1}{2}\left\|U^{N}(t)\right\|_{L^{2}}^{2} \quad \text { (kinetic part) } \\
& E_{N}^{p}(t):=\frac{1}{2}\left\|\Delta W^{N}(t)\right\|^{2}+\frac{1}{2}\left\|\Theta^{N}(t)\right\|^{2}+\frac{a}{4}\left\|\Delta W^{N}\right\|^{4} \quad \text { (potential part). }
\end{aligned}
$$

We split the proof of Theorem 3.1 into a series of lemmas. In the first two lemmas we estimate the kinetic and potential parts of the energy separately. Let $T>0$ be arbitrary.

Lemma 3.2 (Recovery of kinetic energy). Let $n \leq 3$ and $\left(W^{N}, U^{N}, \Theta^{N}\right)$ be a solution of (2.2)-(2.3). Then for every $\epsilon>0$

$$
\begin{aligned}
\int_{0}^{T} E_{N}^{k}(t) d t \leq \lambda_{1}^{-1}\left(E_{N}(0)+E_{N}(T)\right)+4 & \int_{0}^{T} E_{N}(t) d t \\
& +\frac{C^{2} E^{1 / 2}(0) a^{2}}{2 \epsilon} \int_{0}^{T}\left\|\nabla \Theta^{N}\right\|_{L^{2}}^{2} d t,
\end{aligned}
$$

where $C$ is the Sobolev constant of the embedding $H^{1}(\Omega) \subset L^{4}(\Omega)$.

Proof. Note that $M_{1}\left(W^{N}(t), U^{N}(t), \Theta^{N}(t)\right) \in V^{N}$. From the second equation of (2.2) it follows (after integrating by parts in time) that

$$
\begin{aligned}
0= & -\left\langle\Delta^{-1} \Theta^{N}(T), U^{N}(T)\right\rangle+\left\langle\Delta^{-1} \Theta^{N}(0), U^{N}(0)\right\rangle+\int_{0}^{T}\left\langle\Delta^{-1} \Theta_{t}^{N}(t), U^{N}(t)\right\rangle d t \\
& -\int_{0}^{T}\left(\left\langle\Delta^{-1} \Theta^{N}(t), \Delta^{2} W^{N}(t)\right\rangle-\left\langle\Delta^{-1} \Theta^{N}(t), \Delta \Theta^{N}(t)\right\rangle\right. \\
& \left.+a\left\langle\Delta^{-1} \Theta^{N}(t), \Delta\left(\left(\Delta W^{N}(t)\right)^{3}\right)\right\rangle\right) d t .
\end{aligned}
$$

By Green's theorem, this implies

$$
\begin{aligned}
0= & -\left\langle\Delta^{-1} \Theta^{N}(T), U^{N}(T)\right\rangle+\left\langle\Delta^{-1} \Theta^{N}(0), U^{N}(0)\right\rangle+\int_{0}^{T}\left\langle\Delta^{-1} \Theta_{t}^{N}(t), U^{N}(t)\right\rangle d t \\
& -\int_{0}^{T}\left(\left\langle\Theta^{N}(t), \Delta W^{N}(t)\right\rangle-\left\|\Theta^{N}(t)\right\|_{L^{2}}^{2}\right. \\
& \left.+a\left\langle\Theta^{N}(t),\left(\Delta W^{N}(t)\right)^{3}\right\rangle\right) d t .
\end{aligned}
$$

By the last equation in (2.2), and since $V^{N}$ is invariant under $\Delta^{-1}$, we have

$$
0=\left\langle\Delta^{-1} \Theta_{t}^{N}(t)-\Theta^{N}(t)+U^{N}(t), U^{N}(t)\right\rangle .
$$


Equations (3.3) and (3.4) together imply

$$
\begin{aligned}
0= & -\left\langle\Delta^{-1} \Theta^{N}(T), U^{N}(T)\right\rangle+\left\langle\Delta^{-1} \Theta^{N}(0), U^{N}(0)\right\rangle \\
& +\int_{0}^{T}\left(\left\langle\Theta^{N}(t), U^{N}(t)\right\rangle-\left\|U^{N}(t)\right\|_{L^{2}}^{2}\right) d t \\
& -\int_{0}^{T}\left(\left\langle\Theta^{N}(t), \Delta W^{N}(t)\right\rangle-\left\|\Theta^{N}(t)\right\|_{L^{2}}^{2}+a\left\langle\Theta^{N}(t),\left(\Delta W^{N}(t)\right)^{3}\right\rangle\right) d t .
\end{aligned}
$$

Then for every $\epsilon>0$ we have

$$
\begin{aligned}
\int_{0}^{T}\left\|U^{N}(t)\right\|_{L^{2}}^{2} d t-\left(\int_{0}^{T}\left\|\Theta^{N}(t)\right\|_{L^{2}}^{2} d t\right)^{1 / 2}\left(\int_{0}^{T}\left\|U^{N}(t)\right\|_{L^{2}}^{2} d t\right)^{1 / 2} \\
\leq \lambda_{1}^{-1}\left(E_{N}(0)+E_{N}(T)\right)+\epsilon \int_{0}^{T}\left\|\Delta W^{N}(t)\right\|_{L^{2}}^{2} d t \\
\quad+\left(1+\frac{1}{4 \epsilon}\right) \int_{0}^{T}\left\|\Theta^{N}(t)\right\|_{L^{2}}^{2} d t-a \int_{0}^{T}\left\langle\Theta^{N}(t),\left(\Delta W^{N}(t)\right)^{3}\right\rangle d t
\end{aligned}
$$

It now follows that

$$
\begin{aligned}
\int_{0}^{T}\left\|U^{N}(t)\right\|_{L^{2}}^{2} d t \leq & 2 \lambda_{1}^{-1}\left(E_{N}(0)+E_{N}(T)\right)+2 \epsilon \int_{0}^{T}\left\|\Delta W^{N}(t)\right\|_{L^{2}}^{2} d t \\
& +\lambda_{1}^{-1}\left(3+\frac{1}{2 \epsilon}\right) \int_{0}^{T}\left\|\nabla \Theta^{N}(t)\right\|_{L^{2}}^{2} d t \\
& -2 a \int_{0}^{T}\left\langle\Theta^{N}(t),\left(\Delta W^{N}(t)\right)^{3}\right\rangle d t
\end{aligned}
$$

It remains to estimate the nonlinear term. We have by the Hölder inequality, the Sobolev embedding $H^{1}(\Omega) \subset L^{4}(\Omega)$, and the energy inequality,

$$
\begin{aligned}
\int_{0}^{T}\left|\left\langle\Theta^{N}(t),\left(\Delta W^{N}(t)\right)^{3}\right\rangle\right| d t & \leq \int_{0}^{T}\left\|\Theta^{N}(t)\right\|_{L^{4}}\left\|\Delta W^{N}(t)\right\|_{L^{4}}^{3} d t \\
\leq & C \int_{0}^{T}\left\|\Delta W^{N}(t)\right\|_{L^{4}}^{3}\left\|\nabla \Theta^{N}(t)\right\|_{L^{2}} d t \\
\leq & C\left(\int_{0}^{T}\left\|\Delta W^{N}(t)\right\|_{L^{4}}^{6} d t\right)^{1 / 2} \\
& \times\left(\int_{0}^{T}\left\|\nabla \Theta^{N}(t)\right\|_{L^{2}}^{2} d t\right)^{1 / 2} \\
\leq & C E^{1 / 4}(0)\left(\int_{0}^{T}\left\|\Delta W^{N}(t)\right\|_{L^{2}}^{4}\right)^{1 / 2}
\end{aligned}
$$




$$
\begin{aligned}
& \times\left(\int_{0}^{T}\left\|\nabla \Theta^{N}(t)\right\|_{L^{2}}^{2} d t\right)^{1 / 2} \\
\leq & \frac{\epsilon}{a} \int_{0}^{T} E_{N}(t) d t \\
& +\frac{C^{2} E^{1 / 2}(0) a}{4 \epsilon} \int_{0}^{T}\left\|\nabla \Theta^{N}(t)\right\|_{L^{2}}^{2} d t .
\end{aligned}
$$

The statement of the lemma now follows from (3.5) and (3.6).

Lemma 3.3 (Recovery of potential energy). Let $\left(W^{N}, U^{N}, \Theta^{N}\right)$ be a solution of (1.4)-(1.6). Then

$$
\int_{0}^{T} E_{N}^{p}(t) d t \leq \frac{1}{\lambda_{1}}\left(E_{N}(0)+E_{N}(T)\right)+2 \int_{0}^{T} E_{N}^{k}(t) d t+\frac{1}{2 \lambda_{1}} \int_{0}^{T}\left\|\nabla \Theta^{N}\right\|_{L^{2}}^{2} d t .
$$

Proof. Clearly

$$
\int_{0}^{T} E_{N}^{p}(t) d t \leq \frac{1}{2} \int_{0}^{T}\left(\left\|\Delta W^{N}(t)\right\|_{L^{2}}^{2}+a\left\|\Delta W^{N}\right\|_{L^{4}}^{4}+\left\|\Theta^{N}\right\|_{L^{2}}^{2}\right) d t .
$$

We multiply the second equation of (2.2) by the coefficients of $M_{2}\left(W^{N}(t), U^{N}(t)\right.$, $\Theta^{N}(t)$ ), sum from $m=1$ to $N$, and integrate from 0 to $T$. After integrating by parts in $t$, and adding the term $\int_{0}^{T}\left\|\Theta^{N}(t)\right\|_{L^{2}}^{2} d t$ to both sides of the equation, we obtain

$$
\begin{aligned}
& \int_{0}^{T}\left(\left\|\Delta W^{N}(t)\right\|_{L^{2}}^{2}+a\left\|\Delta W^{N}(t)\right\|_{L^{4}}^{4}+\left\|\Theta^{N}(t)\right\|_{L^{2}}^{2}\right) d t \\
&=-W^{N}(T) U^{N}(T)+W^{N}(0) U^{N}(0)+\int_{0}^{T}\left(\left\|U^{N}(t)\right\|_{L^{2}}^{2}\right. \\
&\left.\quad+\left\langle\Theta^{N}(t), \Delta W^{N}(t)\right\rangle+\left\|\Theta^{N}(t)\right\|_{L^{2}}^{2}\right) d t \\
& \leq \lambda_{1}^{-1}\left(E_{N}(0)+E_{N}(T)\right) \\
&+\int_{0}^{T}\left(\left\|U^{N}(t)\right\|_{L^{2}}^{2}+\epsilon\left\|\Delta W^{N}(t)\right\|^{2}+\frac{1}{4 \lambda_{1} \epsilon}\left\|\nabla \Theta^{N}(t)\right\|^{2}\right) d t,
\end{aligned}
$$

where $\epsilon>0$ is arbitrary. Since $\left\|\Delta W^{N}(t)\right\|_{L^{2}}^{2} \leq 2 E_{N}^{p}(t)$, it follows that

$$
\begin{aligned}
(2-2 \epsilon) & \int_{0}^{T} E_{N}^{p}(t) d t \\
& \leq \lambda_{1}^{-1}\left(E_{N}(0)+E_{N}(T)\right)+\int_{0}^{T}\left(\left\|U^{N}(t)\right\|_{L^{2}}^{2}+\frac{1}{4 \lambda_{1} \epsilon}\left\|\nabla \Theta^{N}(t)\right\|^{2}\right) d t,
\end{aligned}
$$

and by choosing $\epsilon=1 / 2$, the result follows. 
Proof of Theorem 3.1. By combining the estimates of Lemma 3.2 and Lemma 3.3, we obtain

$$
\begin{aligned}
\int_{0}^{T} E_{N}(t) d t \leq & \frac{3}{\lambda_{1}}\left(E_{N}(0)+E_{N}(T)\right)+8 \epsilon \int_{0}^{T} E_{N}(t) d t \\
& +\left(\frac{C^{2} \sqrt{E(0)} a^{2}}{\epsilon}+\frac{1}{2 \lambda_{1}}\right) \int_{0}^{T}\left\|\nabla \Theta^{N}(t)\right\|_{L^{2}}^{2} d t,
\end{aligned}
$$

and so by choosing $\epsilon=1 / 16$ we have

$\int_{0}^{T} E_{N}(t) d t \leq \frac{6}{\lambda_{1}}\left(E_{N}(0)+E_{N}(T)\right)+\left(32 C^{2} \sqrt{E(0)} a^{2}+\frac{1}{\lambda_{1}}\right) \int_{0}^{T}\left\|\nabla \Theta^{N}(t)\right\|^{2} d t$.

From the energy identity (3.1), we estimate $E_{N}(0)$ in terms of $E_{N}(T)$ and in terms of the damping which, in turn, leads to:

$$
\int_{0}^{T} E_{N}(t) d t \leq \frac{12}{\lambda_{1}} E_{N}(T)+\left(32 C^{2} \sqrt{E(0)} a^{2}+\frac{7}{\lambda_{1}}\right) \int_{0}^{T}\left\|\nabla \Theta^{N}(t)\right\|^{2} d t .
$$

Since $E_{N}(t) \geq E_{N}(T)$ for $t<T$,

$$
T E_{N}(T) \leq \frac{12}{\lambda_{1}} E_{N}(T)+\left(32 C^{2} \sqrt{E(0)} a^{2}+\frac{7}{\lambda_{1}}\right) \int_{0}^{T}\left\|\nabla \Theta^{N}(t)\right\|^{2} d t .
$$

Hence

$$
\left(T-\frac{12}{\lambda_{1}}\right) E_{N}(T) \leq\left(32 C^{2} \sqrt{E(0)} a^{2}+7 \lambda_{1}^{-1}\right) \int_{0}^{T}\left\|\nabla \Theta^{N}(t)\right\|_{L^{2}}^{2} d t .
$$

Defining

$$
K(s):=\frac{32 C_{\Omega}^{2} \sqrt{s} a^{2}+7 \lambda_{1}^{-1}}{T-6 \lambda^{-1}}
$$

and by choosing $T>6 / \lambda_{1}$, we have

$$
E_{N}(T) \leq K(E(0)) \int_{0}^{T}\left\|\nabla \Theta^{N}(t)\right\|_{L^{2}}^{2} d t .
$$

So by (3.1),

$$
(1+K(E(0))) E_{N}(T) \leq K(E(0)) E_{N}(0)
$$

Consequently,

$$
E_{N}(T) \leq \frac{K(E(0))}{1+K(E(0)} E_{N}(0)=\gamma_{E(0)} E_{N}(0)
$$

where

$$
\gamma_{s}:=\frac{K(s)}{1+K(s)}<1
$$

Propagating the above estimate over the intervals $[k T,(k+1) T], k=1,2, \ldots$, we obtain

$$
E_{N}((k+1) T) \leq \frac{K(E(T))}{1+K(E(k T))} E_{N}(k T)=\gamma_{E(k T)} E_{N}(k T)
$$


Now we notice that $\gamma_{s}$ is an increasing function of $s$. Indeed, this follows from the definition of $\gamma_{s}$ and the fact that $K(s)$ is also an increasing function of $s$. Thus $\gamma_{s_{1}} \leq \gamma_{s_{2}}$, whenever $s_{1} \leq s_{2}$. Since the energy $E(k T)$ is decreasing in $k$, we obtain that

Thus

$$
\gamma_{E(k T)} \leq \gamma_{E(0)} \quad \text { for all } \quad k=1,2 \ldots
$$

This yields

$$
E_{N}((k+1) T) \leq \gamma_{E(0)} E_{N}(k T), \quad k=1,2 \ldots
$$

$$
E_{N}(k T) \leq \gamma_{E(0)}^{k} E_{N}(0), \quad k=0,1,2 \ldots .
$$

Since $\gamma_{E(0)}<1$, one obtains $\gamma_{R}^{k} \leq e^{-\omega_{R} k T}$ with

$$
\omega_{R}=-\frac{\log \gamma_{R}}{T}>0 .
$$

Thus, for $t \in[k T,(k+1) T]$ we have

$$
E(t) \leq E(T k) \leq E(0) e^{-\omega_{R} k T} \leq C E(0) e^{-\omega_{R} t}
$$

where the constant $C$ satisfies:

$$
C=e^{\omega_{R} T} \leq \frac{T+\lambda_{1}^{-1}}{7 \lambda_{1}^{-1}} .
$$

This gives the final conclusion in the statement of the theorem.

\subsection{Exponential stability of the PDE solutions}

We observe that the same proof as that of Theorem 3.1 works for any smooth solution by using the multipliers $-\Delta^{-1} \Theta$ and $W$ in place of $\Delta^{-1} \Theta^{N}$ and $W^{N}$, respectively. Since we have not proved the existence of smooth solutions of the system (1.1)-(1.3), we use the Galerkin approximations and weak convergence to prove exponential decay of the weak solution.

Proof of Theorem 1.5. We begin by observing that using the estimates in Theorem 3.1, we can rework the argument in Subsection 2.7 so that we have the existence of a weak solution for all $t \geq 0$.

From the proof of Theorem 1.3 it follows that

$$
\begin{gathered}
W^{N} \rightarrow W \quad \text { in } \quad L^{2}\left([0, T] ; W^{2,2}(\Omega)\right), \\
U^{N} \rightarrow U \quad \text { in } \quad L^{2}\left([0, T] ; L^{2}(\Omega)\right), \\
\Theta^{N} \rightarrow \Theta \quad \text { in } \quad L^{2}\left([0, T] ; L^{2}(\Omega)\right), \\
\Delta W^{N} \rightarrow \Delta W \quad \text { in } \quad L^{2}\left([0, T] ; L^{4}(\Omega)\right) .
\end{gathered}
$$

Take $[t, t+h] \subset[0, \infty)$. The norm in $L^{2}\left([t, t+h] ; L^{4}(\Omega)\right)$ is weakly lower semicontinuous. Hence

$$
\int_{t}^{t+h} E(s) d s \leq \liminf _{N \rightarrow \infty} \int_{t}^{t+h} E_{N}(s) d s .
$$


So by Theorem 3.1,

$$
\frac{1}{h} \int_{t}^{t+h} E(s) d s \leq \frac{C E(0)}{h} \int_{t}^{t+h} e^{-\omega_{R} s} d s,
$$

where we have used $E_{N}(0) \leq E(0)$. Then the limit as $h \rightarrow 0$ of the left-hand side of (3.16) exists for almost all $t \geq 0$ [50, Theorem 7.11, p. 141]. Consequently, for almost all $t \geq 0, E(t) \leq C E(0) e^{-\omega_{R} t}$.

\subsection{Proof of the corollary}

We need to show that the decay rates $\omega_{R}$ can be made independent on $R$. Our starting point is the estimate in Theorem 1.5. Let $E(0) \leq R$ and let $r>0$ be an arbitrary positive constant. The constant $C$ is given in (1.8) and is independent on $R$. We select the entrance time $t_{R}$ when the solution enters the ball $B(0, r)$. This implies $E\left(t_{R}\right) \leq C R e^{-\omega_{R} t_{R}} \leq r$, hence $t_{R}=\max \left\{0,-\frac{\log \frac{r}{C R}}{\omega_{R}}\right\}$. With the above notation the semigroup property and the estimate (1.8) imply

$$
E(t) \leq C r e^{-\omega_{r}\left(t-t_{R}\right)}, \quad t \geq t_{R} .
$$

The dissipativity relation yields $E(t) \leq R$ for $t \geq 0$, and hence the following cumulative estimate holds:

$$
E(t) \leq \max \{R, C r\} e^{\omega_{r} t_{R}} e^{-\omega_{r} t}=C(R, r) e^{-\omega_{r} t} .
$$

We also recall that

$$
\omega_{R}=-\frac{1}{T} \log \gamma_{R}=-\frac{1}{T} \log \frac{K(R)}{1+K(R)}
$$

where $K(R)$ is given by (3.10). Maximizing $\omega_{r}$ with respect to $r$ leads to taking $r=0$ in $K(r)$, given by $(3.10)$, and gives

$$
\omega^{0}=\sup _{R>0} \omega_{R}=\frac{1}{T} \log \left(\frac{T+\lambda_{1}^{-1}}{7 \lambda_{1}^{-1}}\right) .
$$

Since $C(R, r) \rightarrow \infty$ when $r \rightarrow 0$ we take a suboptimal value of $s=\epsilon^{2}$ which then leads to

$$
\omega_{\epsilon}=\frac{1}{T} \log \left(\frac{T+\lambda_{1}^{-1}+32 C_{\Omega}^{2} a^{2} \epsilon}{7 \lambda_{1}^{-1}+32 C_{\Omega}^{2} a^{2} \epsilon}\right) .
$$

We clearly have $\omega_{\epsilon} \rightarrow \omega_{0}$ as $\epsilon \rightarrow 0$.

Optimizing the above expression with respect to the choice of time $T \in$ $\left(6 \lambda_{1}^{-1}, \infty\right)$ yields the optimal value $T^{0}$ given by

$$
\frac{T^{0}+c}{b}=e^{\frac{T b}{T_{0}+c}},
$$

where $b:=7 \lambda_{1}^{-1}+32 C_{\Omega}^{2} a^{2} \epsilon$ and $c:=\lambda_{1}^{-1}+32 C_{\Omega}^{2} a^{2} \epsilon$. 
Summing up, the "optimal" decay rates are given by $\omega_{\epsilon}^{0}$ given by expression (3.17) with $T$ replaced by $T_{0}$ resulting from solving (3.18). The constant $C(R, \epsilon)$ is estimated by

$$
C(R, \epsilon)=R e^{\omega_{\epsilon} t_{R}}=R e^{\omega_{\epsilon} \omega_{R}^{-1} \log \left(C R \epsilon^{2}\right)}=R\left(\frac{C R}{\epsilon^{-2}}\right)^{\omega_{\epsilon} \omega_{R}^{-1}},
$$

which explicitly computed gives

$$
\omega_{\epsilon} \omega_{R}^{-1}=\log \frac{T_{0}+\lambda_{1}^{-1}+32 C_{\Omega}^{2} \epsilon a^{2}}{7 \lambda_{1}^{-1}+32 C_{\Omega}^{2} \epsilon a^{2}} \times\left(\log \frac{T_{0}+\lambda_{1}^{-1}+32 C_{\Omega}^{2} \sqrt{R} a^{2}}{7 \lambda_{1}^{-1}+32 C_{\Omega}^{2} \sqrt{R} a^{2}}\right)^{-1} .
$$

Clearly, $C(R, \epsilon) \rightarrow \infty$ when $\epsilon \rightarrow 0$. The proof of the corollary is completed.

\section{Appendix}

In this section we shall present results pertaining to existence of smooth solutions corresponding to the nonlinear problem (1.1)-(1.3). This is based on an approach which is very different from the one used before in this paper and leading to weak and global solutions. This approach relies on an explicit use of the analyticity of the semigroup corresponding to the linear part of the model. A critical role is played by the estimates reflecting "maximal regularity" of solutions to non-autonomuos abstract parabolic equations $[1,44]$. The resulting theory will lead to either local in time, or global and small data existence and uniqueness of smooth (classical) solutions, where the smoothness is measured with respect to Hölder continuity. The result obtained is as follows:

Theorem 4.1. With reference to the problem (1.1)-(1.3).

$$
\text { Let } x(0):=\left(\Delta W(0), W_{t}(0), \Theta(0)\right) \in X_{1} \equiv C^{1+\epsilon}(\bar{\Omega}) \times C^{1+\epsilon}(\bar{\Omega}) \times C^{1+\epsilon}(\bar{\Omega}) \text {, }
$$

where $\epsilon>0$ is arbitrary. Then there exists a time $T_{0}>0$ such that $x(t)=$ $(\Delta W(t), U(t), \Theta(t))$ is a unique classical solution satisfying

(1) $(\Delta W, U, \Theta) \in\left[C\left(\left[0, T_{0}\right] \times \bar{\Omega}\right)\right]^{3} \cap\left[C^{1}\left(\left(0, T_{0}\right] \times \bar{\Omega}\right)\right]^{3}$

(2) $\left(\Delta^{2} W, \Delta U, \Delta \Theta\right) \in\left[C\left(\left(0, T_{0}\right] \times \bar{\Omega}\right)\right]^{3}$.

If in addition, $(\Delta W(0), U(0), \Theta(0)) \in\left[C_{0}^{2}(\bar{\Omega})\right]^{3}$, the the solution is strict, and the regularity described above extends to the closed intervals $\left[0, T_{0}\right]$.

The same conclusion holds with an arbitrary $T_{0}<\infty$, provided however that $|x(0)|_{X_{1}} \leq r$, for $r$ sufficiently small.

Remark 4.2. Theorem 4.1 establishes existence and uniqueness of classical solutions defined on some interval $\left[0, T_{0}\right]$. Instead of having solutions local in time one could also obtain, by following the methods in [44] solutions that are global in time at the expense of restricting the analysis to suitably small data. One way or another, this type of result pertains only to local (in time or space) solutions. This is in contrast with Theorem 1.3, which asserts global solutions, however of limited regularity. Combining both types of results with the aim of obtaining a full scale of spaces with various degrees of regularity, appears to be an open and difficult 
problem. The technicalities and methodology involved in the two approaches are very different and incompatible.

Proof. Step 1. Abstract parabolic problem and maximal regularity. We shall first represent the original PDE system (1.1)-(1.3) as an abstract parabolic system. To accomplish this we define $[39,43] Z:=\Delta W$ and set $x:=(Z, U, \theta)$. The differential operator $\Delta$, equipped with zero Dirichlet boundary conditions, generates an analytic semigroup on $L^{2}(\Omega)$. With the above notation, the original system can be written in the following operator form:

$$
x_{t}=\Delta\left[\begin{array}{ccc}
0 & 1 & 0 \\
-1 & 0 & 1 \\
0 & -1 & 1
\end{array}\right] x-a \Delta\left[\begin{array}{c}
0 \\
\phi(Z) \\
0
\end{array}\right]
$$

where $\phi(s):=s^{3}$. Denoting

$$
A:=\Delta\left[\begin{array}{ccc}
0 & 1 & 0 \\
-1 & 0 & 1 \\
0 & -1 & 1
\end{array}\right],
$$

it is easily seen that $A$ is the generator of an analytic semigroup on $H:=L^{2}(\Omega) \times$ $L^{2}(\Omega) \times L^{2}(\Omega)$ and (4.1) can be rewritten as

$$
x_{t}=A x+A F(x)
$$

where

$$
F(x):=-a\left[\begin{array}{lll}
\phi(Z) & 0 & 0
\end{array}\right]^{\top} .
$$

Equation (4.3) is a nonlinear abstract parabolic system defined on $H$. The nonlinearity enters via the generator $A$, and so solvability of the system must depend on "maximal regularity" properties $[16,44]$. Since maximal regularity does not hold within the context of the $L^{\infty}([0, T] ; H)$-topology [44], one should consider the problem within the framework of interpolation spaces based on the $C(\bar{\Omega})$-topology. To accomplish this, we shall adopt and follow the framework of [44].

First of all we will be considering $\Delta: \operatorname{Dom}(\Delta) \subset C(\bar{\Omega}) \rightarrow C(\bar{\Omega})$ with

$$
\operatorname{Dom}(\Delta)=\{\phi \in C(\bar{\Omega}), \Delta \phi \in C(\bar{\Omega}), \phi=0 \text { on } \partial \Omega\} .
$$

Moreover $\operatorname{Dom}(\Delta) \supset\left\{\phi \in C^{2}(\bar{\Omega}), \phi=0\right.$ on $\left.\partial \Omega\right\}$. It is known [44] that $\Delta$ generates an analytic semigroup on $C(\bar{\Omega})$. However, the generator has peculiar properties that include:

(1) it is not densely defined,

(2) it is not strongly continuous.

The operator $A$, whose action is defined in (4.2) is also an extended generator of an analytic semigroup on

$$
X:=C(\bar{\Omega}) \times C(\bar{\Omega}) \times C(\bar{\Omega})
$$

with $\operatorname{Dom}(A):=\operatorname{Dom}(\Delta) \times \operatorname{Dom}(\Delta) \times \operatorname{Dom}(\Delta)$. 
Step 2. Representation as a quasilinear abstract parabolic system. Rewriting

$$
\Delta \phi(u)=\phi^{\prime}(u) \Delta u+\phi^{\prime \prime}(u)|\nabla u|^{2},
$$

we obtain from (4.3) that

$$
x_{t}=A x-a\left[0, \phi^{\prime}(Z) \Delta Z+\phi^{\prime \prime}(Z)|\nabla Z|^{2}, \quad 0\right]^{\top} .
$$

Denoting

$$
A(t, x)=A(x):=A-a\left[\begin{array}{ccc}
0 & 0 & 0 \\
\phi^{\prime}(Z) \Delta & 0 & 0 \\
0 & 0 & 0
\end{array}\right]^{\top}
$$

leads us to the consideration of a quasilinear system :

$$
x_{t}=A(x) x+f(x),
$$

where

$$
f(x) \equiv-a\left[\begin{array}{cc}
0 & \phi^{\prime \prime}(Z)|\nabla Z|^{2} \quad 0
\end{array}\right]^{\top} .
$$

Equation (4.5) is a quasilinear abstract parabolic system studied in [44]. In fact, Theorem 2.1 in [45] gives local existence and uniqueness of solutions under several hypotheses imposed on $A(x)$ and $f(x)$.

Step 3. Verification of the hypotheses for Theorem 2.1. We shall use the notation from [45]. The standing hypotheses (i) and (ii) on page 397 require that for any open set $U \in D_{A}(\theta, \infty)[16,44]$, with some $\theta \in(0,1), A(x): U \rightarrow L(D, X)$ and $f(x)$ are locally Lipschitz with respect to $x \in B\left(x_{0}, r\right) \subset D_{A}(\theta, \infty)$. This is to say,

$$
\| A(x)-\left.A(y)\right|_{L(D, X)}+|f(x)-f(y)|_{X} \leq K|x-y|_{D_{A}(\theta, \infty)}
$$

for $x, y \in B\left(x_{0}, r\right) \subset D_{A}(\theta, \infty)$.

The above is satisfied by taking $\theta>\frac{1}{2}$. For this it suffices to note the presence of $\nabla$ in the definition of $f$ and the characterization [45]

$$
D_{A}(\infty, \theta) \sim\left\{x \in\left[C^{2 \theta}(\bar{\Omega})\right]^{3} \mid x=0 \quad \text { on } \quad \partial \Omega\right\} \quad \text { for } \quad \theta \neq 1 / 2 .
$$

Requirement (ii) in Theorem 2.1 is that $A\left(x_{0}\right)$ for $x_{0} \in U$ is a generator of an analytic semigroup on $X$. This follows from the fact that $A(x)$ is strongly elliptic on the strength of $\left.\phi^{\prime} \geq 0\right)$. Indeed, the operator $A(x)$ can be written as

$$
A\left(x_{0}\right)=\left[I-a \phi^{\prime}\left(Z_{0}\right) M\right] A
$$

where the matrix $M$ is equal to

$$
M \equiv\left[\begin{array}{ccc}
-1 & 1 & -1 \\
0 & 0 & 0 \\
0 & 0 & 0
\end{array}\right]
$$

It is easy to see that for each $x_{0}=\left(Z_{0}, U_{0}, \theta_{0}\right) \in U$ the matrix $I-a \phi^{\prime}\left(Z_{0}\right) M$ is non-singular on the strength of $\phi(Z) \geq 0$. Since $A$ generates an analytic semigroup, so does $A\left(x_{0}\right)$. 
Thus [45, Theorem 2.] gives local existence and uniqueness of classical solutions for any initial data $x(0) \in\left[C_{0}^{1+\epsilon}(\overline{\Omega)}]^{3}\right.$. Similarly, stronger regularity of initial datum $x(0) \in \operatorname{Dom}(A) \times \operatorname{Dom}(A) \times \operatorname{Dom}(A)$ implies that the obtained solution is strict. This implies the second part of Theorem 4.1. The third part of the Theorem follows from the fact that $A$ generates an exponentially stable analytic semigroup. Thus, time globality of small data solutions follows from the theory presented in $[46]$.

\section{Acknowledgements}

(1) The first author is indebted to Alessandra Lunardi for very helpful discussions and for pointing out the reference [45] which is used critically in the proof of Theorem 4.1.

(2) The authors wish to express their thanks to one of the referees for suggestions and comments that led to a more precise description of the decay rates as described in Corollary 1.6.

\section{References}

[1] P. Acquistapace and B. Terreni. Some existence and regularity results for abstract non-autonomous parabolic equations, Journal of Mathematical Analysis and Applications 99:9-64, 1984.

[2] S. A. Ambartsumian, M. V. Belubekyan and M. M. Minasyan. On the problem of vibrations of nonlinear elastic electroconductive plates in transverse and longitudinal magnetic fields, International Journal of Nonlinear Mechanics 19:141-149, 1983.

[3] G. Avalos. Exact controllability of a thermoelastic system with control in the thermal component only, Differential and Integral Equations 22:1-15, 2000.

[4] G. Avalos and I. Lasiecka. Exponential stability of a thermoelastic system with free boundary conditions without mechanical dissipation, SIAM Journal of Mathematical Analysis 29:155-182, 1998.

[5] G. Avalos and I. Lasiecka. Exponential stability of a thermoelastic system without dissipation, Rend. Istit. Mat. Univ. Trieste, Special Volume dedicated to memory of P. Grisvard, XXVIII:1-28, 1997.

[6] G. Avalos and I. Lasiecka. On the null-controllability of thermoelastic plates and singularity of the associated minimal energy function, Journal of Mathematical Analysis and its Applications 10:34-61, 2004.

[7] G. Avalos and I. Lasiecka. Asymptotic rates of blow-up for the minimal energy function for the nullcontrollability of thermoelastic plates: The free case, Control Theory of Partial Differential Equations LNPAM, 242:1-49, Chapman and Hall, CRC, 2005.

[8] G. Avalos and I. Lasiecka. Uniform decays in nonlinear thermoelasticity, In Optimal Control, Theory, Methods and Applications Kluwer, 15:1-22, 1998.

[9] G. Y. Bagdasaryan. Vibrations and Stability of Magnetoelastic Systems (in Russian), Yerevan, 1999 
[10] V. Barbu. Nonlinear Semigroups and Differential Equations in Banach Spaces, Noordhoff, Leiden, 1976.

[11] A. Benabdallah and D. Teniou. Exponential decay rates for a full von Karman model with thermal effects, Electronic Journal of Differential Equations, 7:1-13, 1998.

[12] A. Benabdallah and G. Naso. Nullcontrolability of thermoelastic plates, Abstract and Applied Analysis, 7:585-599, 2002.

[13] I. Chueshov and I. Lasiecka. Long-time behaviour of second order evolution equations with nonlinear damping, Memoirs of AMS, no. 912, 2008.

[14] C. Dafermos. On the existence and asymptotic stability of solutions to the equations of nonlinear thermoelasticity, Arch. Rat. Mechanics. Anal. 29:241-271, 1968.

[15] C. Dafermos and L. Hsiao. Development of singularities in solutions of the equations of nonlinear thermoelasticity, Quart. Appl. Math. 44:463-474, 1986.

[16] G. DaPrato and P. Grisvard. Maximal regularity for evolution equations by interpolation and extrapolation, Journal of Functional Analysis 58:107-124, 1984.

[17] R. Denk and R. Racke. $L^{p}$-resolvent estimates and time decay for generalized thermoelastic plate equations, Electronic Journal of Differential Equations, no. 48, 2006.

[18] R. Denk, Y. Shibata and R. Racke. $L^{p}$ theory for the linear thermoelastic plate equations in bounded and exterior domains, Konstanzer Schriften in Mathematik und Informatik, 240, February, 2008.

[19] M. Eller, I. Lasiecka and R. Triggiani. Simultaneous exact-approximate boundary controllability of thermo-elastic plates with variable thermal coefficients and moment control, Journal of Mathematical Analysis and its Applications, 251:452-478, 2000.

[20] M. Eller, I. Lasiecka and R. Triggiani. Unique continuation result for thermoelastic plates, Inverse and Ill-Posed Problems 9:109-148, 2001.

[21] L. C. Evans. Partial Differential Equations, American Mathematical Society, Providence, RI, 1998.

[22] S. Hansen and B. Zhang. Boundary control of a linear thermoelastic beam, Journal of Mathematical Analysis and its Applications, 210:182-205, 1997.

[23] S. Hansen. Exponential decay in a linear thermoelastic rod, J. Math. Anal. Appl. 187:428-442, 1992.

[24] D. Hasanyan, N. Hovakimyan, A. J. Sasane and V. Stepanyan. Analysis of nonlinear thermoelastic plate equations, In Proceedings of the 43rd IEEE Conference on Decision and Control, 2:1514-1519, 2004.

[25] A.A. Ilyushin. Plasticity. Part One. Elasticity-Plastic Deformations (Russian), OGIZ, Moscow-Leningrad, 1948.

[26] S. Jiang and R. Racke. Evolution Equations in Thermoelasticity, Chapman and Hall, Boca Raton, FL, 2000.

[27] J. U. Kim. On the energy decay of a linear thermoelastic bar and plate, SIAM Journal of Mathematical Analysis, 23:889-899, 1992.

[28] H. Koch and I.Lasiecka. Backward uniqueness in linear thermo-elasticity with variable coefficients, Functional Analysis and Evolution Equations, special volume dedicated to G. Lumer, Birkhäuser, 2007.

[29] J. Lagnese. The reachability problem for thermoelastic plates, Archive for Rational Mechanics and Analysis 112:223-267, 1990. 
[30] J. Lagnese. Boundary Stabilization of Thin Plates, SIAM, 1989.

[31] I. Lasiecka and D. Tataru. Uniform boundary stabilization of semilinear wave equations with nonlinear boundary damping, Differential and Integral Equations, 6:507533, 1993.

[32] I. Lasiecka. Uniform decay rates for full von Karman system of dynamic thermoelasticity with free boundary conditions and partial boundary dissipation, Communications in Partial Differential Equations, 24:1801-1847, 1999.

[33] I. Lasiecka and C. Lebiedzik. Asymptotic behavior of nonlinear structural acoustic interactions with thermal effects on the interface, Nonlinear Analysis 49:703-735, 2002 .

[34] I. Lasiecka and C. Lebiedzik. Boundary stabilizability of nonlinear structural acoustic models with thermal effects on the interface, C.R. Acad. Sci. paris t 1. 329:187-192, 2000 .

[35] I. Lasiecka and T. Seidman. Blowup estimates for observability of a thermoelastic system, Asymptotic Analysis 50:93-120, 2006.

[36] I. Lasiecka and R. Triggiani. Structural decomposition of thermoelastic semigroups with rotational forces, Semigroup Forum 60:16-60, 2000.

[37] I. Lasiecka and R. Triggiani. Control Theory for PDEs, vol. 1. Cambridge University Press, 2000

[38] I. Lasiecka, M. Renardy and R. Triggiani. Backward uniqueness of thermoelastic plates with rotational forces, Semigroup Forum 62:217-242, 2001.

[39] I. Lasiecka and R. Triggiani. Exact null-controllability of structurally damped and thermoelastic parabolic models, Rend. Mat. Acta Lincei 9:43-69, 1998.

[40] I. Lasiecka and R. Triggiani. Analyticity, and lack thereof, of thermoelastic semigroups, European Series in Applied and Industrial Mathematics, 4:199-222, 1998.

[41] L. Librescu. Elastostatics and Kinetics of Anisotropic and Heterogeneous Shell-type Structures, Noordhoff, Leiden, 1975.

[42] L. Librescu, D. Hasanyan, Z. Qin and D. Ambur. Nonlinear magnetothermoelasticity of anisotropic plates in a magnetic field, Journal of Thermal Stresses, 26:1277-1304, 2003 .

[43] Z. Liu and M. Renardy. A note on the equation of a thermoelastic plate, Appl. Math. Letters 8:1-6, 1995.

[44] A. Lunardi. Analytic Semigroups and Optimal Regularity in Parabolic problems, Birkhäuser, 1995.

[45] A. Lunardi. Abstract quasilinear parabolic equations, Math. Ann. 267:395-415, 1984.

[46] A. Lunardi. Global solutions of abstract quasilinear parabolic equations, Journal Differential Equations vol. 58, 228-242, 1985.

[47] A. Pazy. Semigroups of Linear Operators and Applications to Partial Differential Equations, Applied Mathematical Sciences, 44, Springer Verlag, New York, 1983.

[48] J. E. Muñoz Rivera and R. Racke. Smoothing properties, decay, and global existence of solutions to nonlinear coupled systems of thermoelastic type, SIAM Journal on Mathematical Analysis no. 6, 26:1547-1563, 1995.

[49] J. E. Muñoz Rivera and R. Racke. Large solutions and smoothing properties for nonlinear thermoelastic systems, Journal of Differential Equations no. 2, 127:454483, 1996. 
[50] W. Rudin. Real and Complex Analysis. Third Edition, McGraw-Hill, New York, 1987.

[51] J. Simon. Compact Sets in the Space $L^{p}(0, T ; B)$, Ann. Mat. Pura Appl. 146:65-96, 1987.

[52] R. Temam. Infinite-Dimensional Dynamical Systems in Mechanics and Physics, 2nd ed. Springer, New York, 1997.

[53] H. Triebel. Interpolation Theory, Function Spaces, Differential Operators, North Holland, 1978.

Irena Lasiecka

Department of Mathematics

University of Virginia

Charlottesville, VA 22903

USA

e-mail: il2v@virginia.edu

Sara Maad

Department of Mathematics

Stockholm University

S-106 91 Stockholm

Sweden

e-mail: maad@math.su.se

Amol Sasane

Department of Mathematics

London School of Economics

Houghton Street

London WC2A 2AE

United Kingdom

e-mail: A.J.Sasane@lse.ac.uk

Received: 23 February 2008.

Revised: 28 June 2008.

Accepted: 16 July 2008. 